\title{
Probing neutrino parameters with a Two-Baseline Beta-beam set-up
}

\section{Sanjib Kumar Agarwalla*†}

Department of Physics, Virginia Tech, Blacksburg, VA 24061, USA

E-mail: sanjib@vt.edu

\section{Sandhya Choubey}

Harish-Chandra Research Institute,

Chhatnag Road, Jhunsi, Allahabad 211019, India

E-mail: sandhya@hri.res.in

\section{Amitava Raychaudhuri}

Harish-Chandra Research Institute,

Chhatnag Road, Jhunsi, Allahabad 211019, India

E-mail: raychaudehri.res.in

We discuss the prospects of exploring the neutrino mass parameters with a CERN based Betabeam experiment using two different detectors at two different baselines. The proposed set-up consists of a $50 \mathrm{kton}$ iron calorimeter (ICAL) at a baseline of around $7150 \mathrm{~km}$ which is roughly the magic baseline, e.g., ICAL@INO, and a 50 kton Totally Active Scintillator Detector at a distance of $730 \mathrm{~km}$, e.g., at Gran Sasso. We take ${ }^{8} \mathrm{~B}$ and ${ }^{8} \mathrm{Li}$ source ions with a boost factor $\gamma$ of 650 for the magic baseline while for the closer detector we consider ${ }^{18} \mathrm{Ne}$ and ${ }^{6} \mathrm{He}$ ions with a range of Lorentz boosts. We find that the locations of the two detectors complement each other leading to an exceptional high sensitivity. With $\gamma=650$ for ${ }^{8} \mathrm{~B} /{ }^{8} \mathrm{Li}$ and $\gamma=575$ for ${ }^{18} \mathrm{Ne} /{ }^{6} \mathrm{He}$ and total luminosity corresponding to $5 \times\left(1.1 \times 10^{19}\right)$ and $5 \times\left(2.9 \times 10^{19}\right)$ useful ion decays in neutrino and antineutrino modes respectively, we find that the two-detector set-up can probe maximal $\mathrm{CP}$ violation and establish the neutrino mass ordering if $\sin ^{2} 2 \theta_{13}$ is $1.8 \times 10^{-5}$ and $4.6 \times 10^{-5}$, respectively, or more. The sensitivity reach for $\sin ^{2} 2 \theta_{13}$ itself is $5.3 \times 10^{-5}$. CP violation can be discovered for $64 \%$ of the possible $\delta_{C P}$ values for $\sin ^{2} 2 \theta_{13} \geq 8 \times 10^{-5}$.

10th International Workshop on Neutrino Factories, Super beams and Beta beams

June 30 - July 52008

Valencia, Spain

\footnotetext{
*Speaker.

$\dagger$ It is my pleasure to acknowledge the support of Harish-Chandra Research Institute (HRI) where the work has been done. The computational work presented in this talk has been performed using the HRI cluster facilities. I would like to thank Anselmo Cervera for providing me the local hospitality during Nufact08.
} 

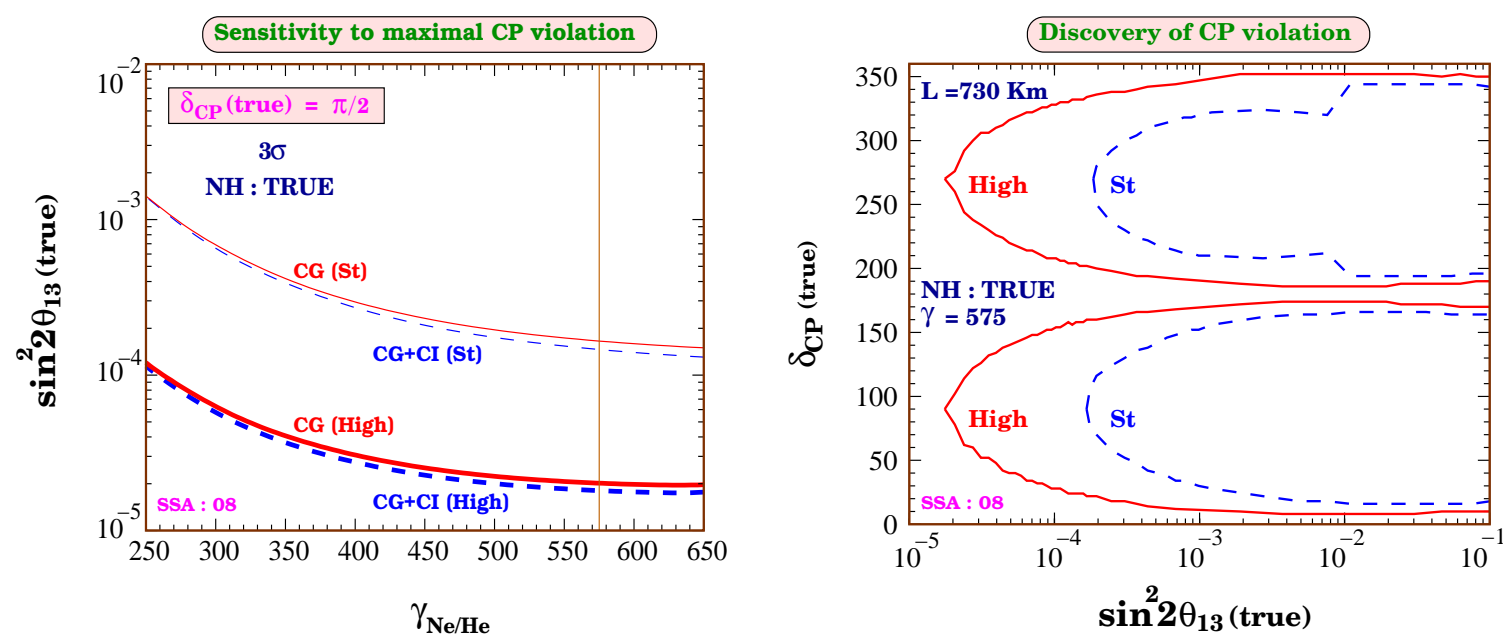

Figure 1: Left panel shows the $3 \sigma \sin ^{2} 2 \theta_{13}$ (true) reach for sensitivity to "maximal CP violation". The red solid curves (marked as 'CG') are for the CERN-TASD@LNGS alone while the blue dashed lines (marked as 'CG+CI') are for the combined data from CERN-TASD@LNGS and CERN-ICAL@ INO. The results are shown as a function of the Lorentz boost for ${ }^{18} \mathrm{Ne}$ and ${ }^{6} \mathrm{He}$ (taken same for both ions), for $\delta_{C P}(\operatorname{true})=90^{\circ}$. Thick lines (marked "High") are for $5 \times\left(1.1 \times 10^{19}\right)$ useful ${ }^{18} \mathrm{Ne}$ and ${ }^{8} \mathrm{~B}$ decays and $5 \times\left(2.9 \times 10^{19}\right)$ useful ${ }^{6} \mathrm{He}$ and ${ }^{8} \mathrm{Li}$ decays, while thin lines (marked "St") are for $5 \times\left(1.1 \times 10^{18}\right)$ and $5 \times\left(2.9 \times 10^{18}\right)$ useful ion decays respectively. In the right panel, the area enclosed by the curves represents the $3 \sigma$ range of $\delta_{C P}($ true $)$ as a function of $\sin ^{2} 2 \theta_{13}$ (true) for which the data can be used to rule out the CP-conserving scenario using the CERN-Gran Sasso reference TASD set-up with ${ }^{18} \mathrm{Ne}$ and ${ }^{6} \mathrm{He}$ as source ions.

We consider a two-baseline Beta-beam [1] set-up, one with $L=7152 \mathrm{~km}$, the CERN-INO baseline [2], and another with $L=730 \mathrm{~km}$ which is the CERN-Gran Sasso (LNGS) distance. For the CERN-INO case ${ }^{8} \mathrm{~B}$ and ${ }^{8} \mathrm{Li}$ are the preferred source ions and we take $\gamma=650$. For the CERNLNGS set-up, on the other hand, we choose the ${ }^{18} \mathrm{Ne}$ and ${ }^{6} \mathrm{He}$ ions and allow their $\gamma$ to vary between 250-650. Since the ${ }^{8} \mathrm{~B}$ and ${ }^{8} \mathrm{Li}$ ions would produce multi-GeV neutrino beams for $\gamma=650$, we use a 50 kton iron calorimeter (ICAL) for the longer baseline at INO [3]. For the intermediate baseline option, since we are interested in the lower energy ${ }^{18} \mathrm{Ne}$ and ${ }^{6} \mathrm{He}$ ions, we assume a $50 \mathrm{kton}$ Totally Active Scintillator Detector (TASD) in order to harness the low energy events required for better CP sensitivity. We present results for $5 \times\left(1.1 \times 10^{19}\right)$ and $5 \times\left(2.9 \times 10^{19}\right)$ useful ion decays in neutrino and antineutrino modes respectively for both baseline set-ups. We also show the projected sensitivity with one order less statistics.

The $3 \sigma$ sensitivity to "maximal CP violation" is presented in Fig. 1. The two-baseline combined results for $\operatorname{sgn}\left(\Delta m_{31}^{2}\right)$ and $\sin ^{2} 2 \theta_{13}$ sensitivity reach have been depicted in Fig. 2.

In conclusion, we present Table 1 containing all the essential results.

\section{References}

[1] P. Coloma et al., JHEP 0805, 050 (2008); S. K. Agarwalla et al., Nucl. Phys. B 805, 305 (2008).

[2] S. K. Agarwalla et al., Phys. Lett. B 629, 33 (2005); S. K. Agarwalla et al., Nucl. Phys. B 771, 1 (2007); S. K. Agarwalla et al., Nucl. Phys. B 798, 124 (2008); S. K. Agarwalla et al., Phys. Rev. D 

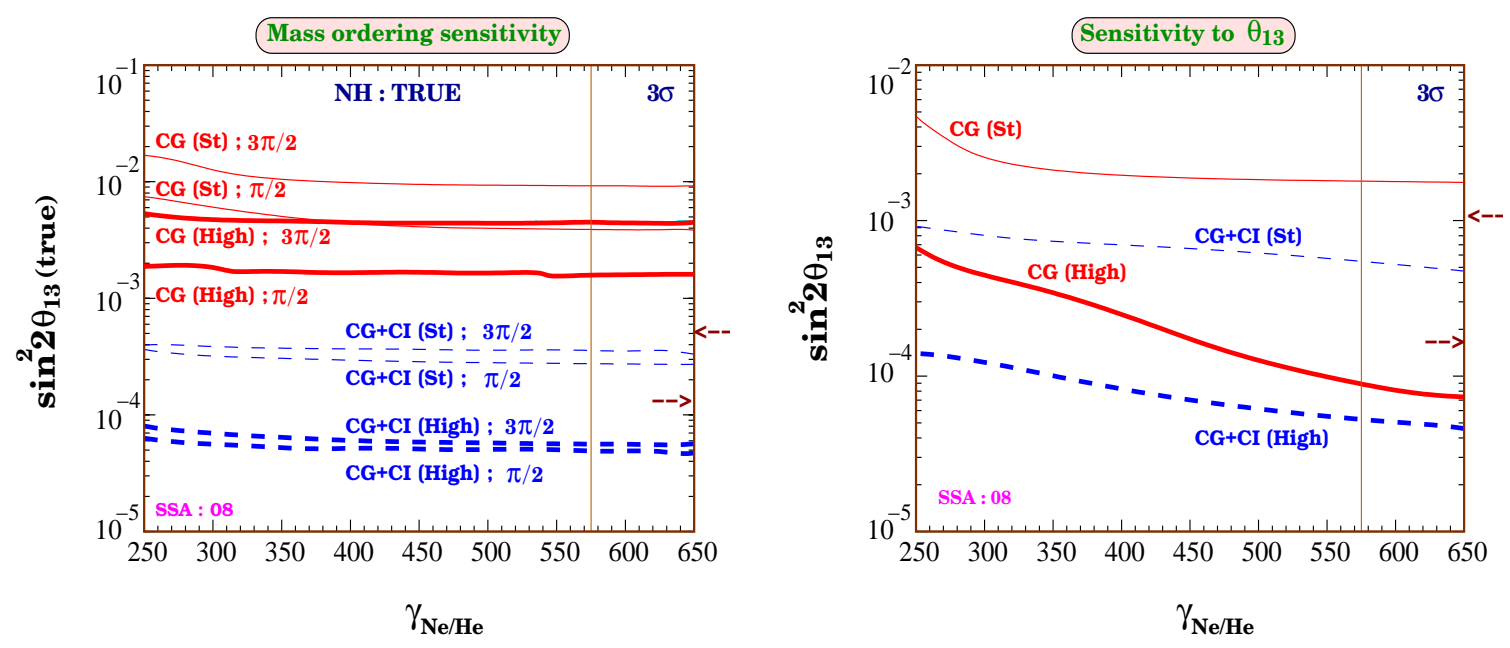

Figure 2: The left and right panels depict the $\operatorname{sgn}\left(\Delta m_{31}^{2}\right)$ sensitivity reach and the $\sin ^{2} 2 \theta_{13}$ sensitivity reach, respectively, at $3 \sigma$ as a function of the boost factor for ${ }^{18} \mathrm{Ne}$ and ${ }^{6} \mathrm{He}$. In both panels, the red solid lines are for CERN-TASD@LNGS alone while the blue dashed lines are for the combined data from CERNICAL@INO and CERN-TASD@LNGS. Results for $\delta_{C P}($ true $)=\pi / 2$ and $3 \pi / 2$ are shown. Thick lines are for $5 \times\left(1.1 \times 10^{19}\right)$ useful ${ }^{18} \mathrm{Ne}$ and ${ }^{8} \mathrm{~B}$ decays and $5 \times\left(2.9 \times 10^{19}\right)$ useful ${ }^{6} \mathrm{He}$ and ${ }^{8} \mathrm{Li}$ decays, while thin lines are for $5 \times\left(1.1 \times 10^{18}\right)$ and $5 \times\left(2.9 \times 10^{18}\right)$ useful ion decays respectively. The sensitivity reaches for the CERN-ICAL@INO set-up alone are indicated for both luminosities (for $\delta_{C P}($ true) $=0$ in the left panel) by arrows on the right side of the panels. The location of $\gamma=575$ is shown.

\begin{tabular}{|c||c||c||c|}
\hline \hline Set-up & $\sin ^{2} 2 \theta_{13}$ Discovery $(3 \sigma)$ & Mass Ordering $(3 \sigma)$ & Maximal CP violation $(3 \sigma)$ \\
\hline \hline $\begin{array}{c}\text { CERN-INO } \\
{ }^{8} \mathrm{~B}+{ }^{8} \mathrm{Li}, \gamma=650\end{array}$ & $9.5 \times 10^{-5}$ & $9.4 \times 10^{-5}$ & Not possible \\
\hline \hline $\begin{array}{c}\mathrm{CERN}-\mathrm{HNGS} \\
{ }^{18} \mathrm{Ne}+{ }^{6} \mathrm{He}, \gamma=575\end{array}$ & $2.07 \times 10^{-5}$ & $1.58 \times 10^{-3}$ & $1.97 \times 10^{-5}$ \\
\hline \hline $\begin{array}{c}\mathrm{CERN}-\mathrm{LNGS} \\
{ }^{18} \mathrm{Ne}+{ }^{6} \mathrm{He}, \gamma=575 \\
+\end{array}$ & & & \\
$\begin{array}{c}\mathrm{CERN}-\mathrm{INO} \\
{ }^{8} \mathrm{~B}+{ }^{8} \mathrm{Li}, \gamma=650\end{array}$ & $1.88 \times 10^{-5}$ & $4.64 \times 10^{-5}$ & $1.78 \times 10^{-5}$ \\
\hline \hline Optimized & & & \\
Neutrino Factory & $1.5 \times 10^{-5}$ & $1.5 \times 10^{-5}$ & $1.5 \times 10^{-5}$ \\
\hline \hline
\end{tabular}

Table 1: Results are shown for a five-year run with the luminosity $1.1 \times 10^{19}\left(2.9 \times 10^{19}\right)$ useful ion decays per year in the $v(\bar{v})$ mode. The numbers correspond to $\delta_{C P}$ (true $)=90^{\circ}$. For comparison, the expectations from an optimized two-baseline Neutrino Factory set-up with upgraded magnetized iron detectors are also listed $[4,5]$.

75, 097302 (2007); S. K. Agarwalla et al., JHEP 0806, 090 (2008); R. Adhikari et al., Phys. Lett. B 642, 111 (2006); S. K. Agarwalla et al., Phys. Lett. B 647, 380 (2007).

[3] See http://www.imsc.res.in/ ino.

[4] A. Bandyopadhyay et al. [ISS Physics Working Group], arXiv:0710.4947 [hep-ph].

[5] P. Huber, M. Lindner, M. Rolinec and W. Winter, Phys. Rev. D 74, 073003 (2006). 